\title{
Space charge effects in bunches for different rf wave forms
}

\author{
Oliver Boine-Frankenheim* \\ Gesellschaft für Schwerionenforschung (GSI), Planckstrasse 1, 64291 Darmstadt, Germany
}

Tripti Shukla

Indian Institute of Technology (IIT), New Dehli, 110016, India

(Received 15 December 2004; published 10 March 2005)

\begin{abstract}
The interplay of nonlinear rf fields and space charge is studied using a particle simulation code together with analytic derivations. In the framework of the elliptic ("Hofmann-Pedersen") distribution function the matched beam parameters are obtained. Using the simulation "Schottky" noise from matched bunches the coherent mode spectrum is analyzed and compared with analytic expression. The bunch response to a small rf phase modulation is studied over a large range of initial simulation parameters (modulation frequency, bunch intensity). These bunch response scans clearly show the location of the dipole mode frequency as well as the threshold for the loss of Landau damping due to space charge. In addition, bunch stability scans are performed in order to determine the stability boundaries for flattopped bunches in single and double rf wave forms. The results are related to previous work on beam transfer functions in single and double rf buckets and to experimental observations in the GSI synchrotron SIS.
\end{abstract}

DOI: 10.1103/PhysRevSTAB.8.034201

\section{INTRODUCTION}

Longitudinal space charge effects play an important role in storage rings or synchrotrons for high current ion beams. The induced effects range from synchrotron tune shifts (see, e.g., [1]) to coherent mode splitting [2] that can both be observed with high accuracy from the Schottky noise spectrum, as demonstrated in the GSI heavy ion cooler storage ring ESR [3].

Below transition space charge reduces the effective rf voltage seen by the beam particles. This usually requires an increase of the applied rf voltage in order to compensate for the reduction of the bucket area. Space charge affects the frequencies and the damping of coherent bunch modes. This in turn changes the bunch instability thresholds and the corresponding impedance budget.

For bunches that are very short relative to the rf wavelength, but still long compared to the beam pipe diameter, it is straightforward to calculate the space charge induced incoherent synchrotron frequency shift (see, e.g., [1]). The space charge induced coherent mode splitting in short bunches was analyzed by Neuffer in Ref. [2] and also by Besnier [4], who included also the effect of a small synchrotron frequency spread due to the nonlinear rf field.

At low or medium beam energies, bunches usually cannot be regarded as being short relative to the rf wave length. Particles with large amplitudes will be affected by the nonlinear components of the $\mathrm{rf}$ field and by space charge. This is especially the case if a second harmonic rf system is employed in order to flatten the bunch profile and to increase the transverse space charge limit (see, e.g.,

\footnotetext{
*Email address: O.Boine-Frankenheim@gsi.de Electronic address: http://www-linux.gsi.de/ boine
}

PACS numbers: 29.27.Bd, 29.20.-c, 52.59.Sa, 52.65.Rr

[5]). In the case of such a double rf system the particle motion is fully nonlinear. A double rf system is foreseen in order to increase the bucket area and also the transverse space charge limit in the GSI synchrotron SIS [6]. Because of the low injection energy $(11.4 \mathrm{MeV} / \mathrm{u})$ and the demand for highest longitudinal beam quality, the intense uranium bunches will be strongly affected by longitudinal space charge.

An excellent review of nonlinear single particle motion in single and double rf wave forms can be found in Ref. [7]. A self-consistent treatment of matched bunches affected by space charge in arbitrary rf wave forms was presented by Hofmann and Pedersen in Ref. [8] and applied to long bunches in a single rf wave. In Ref. [8] an analytic expression for the threshold intensity for the "loss of Landau damping" in the case of the rigid dipole mode in a single rf wave was obtained. For sufficiently high intensities space charge suppresses the decoherence of dipole oscillations, leading to persistent oscillations of intense bunches $[9,10]$. In the present work we apply the theory by Hofmann and Pedersen to double rf systems.

Of particular interest for the study of beam stability thresholds is the response of long bunches to rf phase or amplitude modulations. In Ref. [11] the response of a bunch to small rf phase or amplitude modulations was studied in the framework of the beam transfer function formalism. It was found that long bunches in a double rf wave are intrinsically unstable, because of the vanishing derivative of the synchrotron frequency inside the bunch (but outside the bunch center). In a system with nonmonotonic behavior of the synchrotron frequency, Landau damping can be lost for certain particle amplitudes. Whether the infinite response function obtained in Ref. [11] from linearized Vlasov theory can lead to ob- 
servable effects in high resolution, nonperturbative simulation studies is one of the subjects of the present work. We will focus on the "Hofmann-Pedersen" or elliptic bunch distribution [8]. The stability of Gaussian bunches in a quadratic potential well affected by nonlinear space charge has been studied, e.g., in Ref. [12].

It is important to point out that the effect of rf phase or amplitude modulation on the single particle dynamics has been studied extensively in the literature (see Ref. [7]). In Ref. [13] it was shown that for a modulated rf phase the response of the synchrotron motion in a single rf wave shows characteristics of chaotic particle dynamics in a parametric resonant system. The importance of these findings for the collective response of bunches affected by space charge is not clear.

The paper is organized as follows: In Sec. II we introduce the longitudinal equation of motion, in Sec. III the matched bunch model will be described. Afterward we briefly describe single and double rf waves and the resulting synchrotron motion including space charge (Sec. IV). The rigid dipole mode, the loss of Landau damping, and corresponding observations will be discussed in Secs. V, VI, and VII. After a brief discussion of previous work on beam transfer function (Sec. VIII), we will discuss the results of our simulation studies in Sec. IX. Flattopped bunches and their stability will be discussed in Sec. X.

\section{LONGITUDINAL EQUATION OF MOTION}

Let $z$ and $v$ be the deviation in position and velocity from the synchronous particle. Then the longitudinal equation of motion is

$$
\dot{v}=\frac{1}{m^{*}} \frac{q V}{L}, \quad \dot{z}=v,
$$

with the effective mass $m^{*}=-\gamma_{0} m / \eta$, the relativistic parameter $\gamma_{0}$, the slip factor $\eta=1 / \gamma_{t}^{2}-1 / \gamma_{0}^{2}$, the transition parameter $\gamma_{t}$, the ring circumference $L$, the charge $q$, and the voltage profile $V(z)$. The relation between the $z$ coordinate and the rf phase is given through $\phi=\phi_{s}-$ $n z / R$, with the synchronous phase $\phi_{s}$, the ring radius $R$, and the harmonic number $n$. The momentum spread is related to the velocity through $v=-\eta \beta c(\Delta p / p)$, with the relativistic parameter $\beta$. The equation of motion in the $(z, v)$ coordinates can be derived from the Hamiltonian

$$
H=\frac{v^{2}}{2}-\frac{q V_{0}}{m^{*} L} Y(z)
$$

with the potential

$$
Y(z)=\frac{1}{V_{0}} \int_{0}^{z} V d z
$$

and the voltage amplitude $V_{0}$. The voltage profile can be divided into the external (rf) voltage part and the space charge part

$$
V=V_{\mathrm{rf}}+V_{s}
$$

The space charge voltage is given through (see, e.g., Ref. [1])

$$
V_{s}=2 \pi R E_{s}=-q \beta c R X_{s} \frac{\partial \lambda}{\partial z},
$$

with the space charge electric field $E_{s}$, the line density $\lambda(z)$, the space charge reactance

$$
X_{s}=\frac{g}{2 \epsilon_{0} \beta_{0} c \gamma_{0}^{2}},
$$

and the $g$ factor (see, e.g., Ref. [14]). For the space charge potential one obtains

$$
Y_{s}=q \beta_{0} c \frac{R X_{s}}{V_{0}}\left(\lambda_{0}-\lambda\right)
$$

with the line density $\lambda_{0}$ at $z=0$.

\section{ELLIPTIC BUNCH DISTRIBUTION}

If the Hamiltonian is a constant of motion, any stationary ("matched") distribution function can be written as a function of $H$. The analytic analysis in the presence of space charge can be greatly simplified if a local elliptic (Hofmann-Pedersen) distribution function [8] is assumed,

$$
\begin{gathered}
g(H)=c_{1} \sqrt{H_{m}-\bar{H}}, \\
H_{m}=\frac{v_{m}^{2}}{2}=-\frac{q V_{0}}{m^{*} L} Y\left(z_{m 2}\right),
\end{gathered}
$$

with the normalization constant $c_{1}$, the value of the Hamiltonian $H_{m}$ for the bunch boundary particle, the velocity $v_{m}$ at the bunch center, and the potential $Y\left(z_{m 2}\right)$ at one end of the bunch. For the distribution function in $(z, v)$ space we get

$$
f(v, z)=c_{2} \sqrt{v_{m}^{2}(z)-v^{2}},
$$

with the velocity function for the boundary particle

$$
v_{m}^{2}(z)=\frac{2 q V_{0}}{L m^{*}}\left(Y(z)-Y\left(z_{m 2}\right)\right)
$$

The line density is obtained from Eq. (10) as

$$
\begin{gathered}
\lambda(z)=c_{3}\left(Y(z)-Y\left(z_{m 2}\right)\right)=\frac{N}{u_{m}}\left(Y_{\mathrm{rf}}(z)-Y_{\mathrm{rf}}\left(z_{m 2}\right)\right), \\
u_{m}=\int_{z_{m 1}}^{z_{m 2}}\left(Y_{\mathrm{rf}}(z)-Y_{\mathrm{rf}}\left(z_{m 2}\right)\right) d z,
\end{gathered}
$$

with the number of particles $N$ in the bunch. The bunching factor can be defined as

$$
\frac{1}{B_{f}}=\frac{L}{N} \lambda\left(z_{0}\right)=\frac{L}{u_{m}}\left(Y_{\mathrm{rf}}\left(z_{0}\right)-Y_{\mathrm{rf}}\left(z_{m 2}\right)\right),
$$

assuming that there is just one bunch in the machine. In the 
case of an elliptic distribution function, the space charge potential induced by a bunch is directly proportional to the external potential. The total potential can be written in the form

$$
\begin{gathered}
Y(z)=Y_{\mathrm{rf}}(z)\left(1-\frac{V_{s 0}}{V_{0}}\right), \\
V_{s 0}=\operatorname{sgn}\left(m^{*}\right) q \beta_{0} c R X_{s} \frac{N}{u_{m}},
\end{gathered}
$$

with the space charge voltage amplitude $V_{s 0}$. Below transition $\left(m^{*}>0\right)$ the limiting bunch intensity is given through $V_{s 0}=V_{0}$. At this intensity the external focusing field is exactly canceled by the space charge field. Below this limiting bunch intensity and for bunch boundaries $z_{m 1}, z_{m 2}$ not exceeding the bucket boundaries, the matched voltage amplitude can be obtained as

$$
V_{0}=-\frac{L m^{*} v_{m}^{2}}{2 q Y_{\mathrm{rf}}\left(z_{m}\right)}+V_{s 0}
$$

or

$$
V_{0}=-\frac{\pi R m^{*} v_{m}^{2}}{q Y_{\mathrm{rf}}\left(z_{m 2}\right)}(1+\Sigma),
$$

with the space charge parameter

$$
\Sigma=\frac{1}{V_{0} / V_{s 0}-1}=-\frac{q V_{s 0} Y_{\mathrm{rf}}\left(z_{m 2}\right)}{\pi m^{*} v_{m}^{2} R} .
$$

\section{SYNCHROTRON FREQUENCY IN SINGLE AND DOUBLE RF WAVES}

The theory presented in the foregoing sections is expressed in a general way in order to be applicable also to "exotic" voltage profiles (e.g., barrier waves). However, the applications presented in this work will specialize on nonlinear particle motion in single and double rf waves. In these cases the rf phase coordinate $\phi$ is more appropriate than the $z$ coordinate used in the previous derivations. The voltage profile for a single $(\alpha=0)$ and for a double $(\alpha>$ $0)$ rf system operating also at the second harmonic of the main $\mathrm{rf}$ is given through (see, e.g., [7])

$$
\begin{aligned}
\frac{V(\phi)}{V_{0}}= & \sin (\phi)-\sin \left(\phi_{s}\right)-\alpha\left(\sin \left[\phi_{s 2}+2\left(\phi-\phi_{s}\right)\right]\right. \\
& \left.-\sin \left(\phi_{s 2}\right)\right) .
\end{aligned}
$$

In order to obtain a flattened rf potential well with a double rf system, the first and the second derivative of the voltage profile should vanish at $\phi=\phi_{s}$ [5]. For stationary bunches one obtains $\alpha=0.5$.

The synchrotron period as a function of the left and right particle oscillation amplitudes $\hat{\phi}_{1}, \hat{\phi}_{2}$ can be derived from

$$
T_{s}\left(\hat{\phi}_{2}\right)=2 \frac{R}{n} \int_{\hat{\phi}_{1}}^{\hat{\phi}_{2}} \frac{d \phi}{\hat{v}(\phi)}
$$

with the velocity amplitude function

$$
\hat{v}^{2}(\phi)=\frac{q V_{0}}{\pi n m^{*}}\left(Y(\phi)-Y\left(\hat{\phi}_{2}\right)\right) .
$$

For the elliptic distribution the effect of space charge can be cast into a simple, multiplicative factor

$$
T_{s}=\frac{\sqrt{2}}{\omega_{s 0}} \sqrt{1+\Sigma} \int_{\hat{\phi}_{1}}^{\hat{\phi}_{2}} \frac{d \phi}{\sqrt{\left|Y_{\mathrm{rf}}(\phi)-Y_{\mathrm{rf}}\left(\hat{\phi}_{2}\right)\right|}},
$$

with the small amplitude synchrotron frequency (assuming $\Sigma=0$ and $\alpha=0$ )

$$
\omega_{s 0}^{2}=\frac{q h V_{0}}{R L\left|m^{*}\right|} .
$$

In the case of a single, stationary rf wave the following result for the synchrotron oscillation frequency can be obtained (see, e.g., [7], p. 235, for $\Sigma=0$ ):

$$
\frac{\omega_{s}}{\omega_{s 0}}=\frac{\pi}{2} \sqrt{\frac{1}{1+\Sigma}} \frac{1}{K\left(\sin \frac{\hat{\phi}}{2}\right)},
$$

with the elliptic integral of the first kind $K(x)$. The approximation for small amplitudes yields

$$
\frac{\omega_{s}}{\omega_{s 0}} \approx \sqrt{\frac{1}{1+\Sigma}}\left(1-\frac{\hat{\phi}^{2}}{16}\right) .
$$

For a stationary, double rf wave we obtain the exact expression (see, e.g., [7], p. 301, for $\Sigma=0$ )

$$
\frac{\omega_{s}}{\omega_{s 0}}=\sqrt{\frac{1}{1+\Sigma}} \frac{\pi}{\sqrt{2}} \frac{\sin \frac{\hat{\phi}}{2}}{K\left[\sqrt{\frac{1}{2}\left(1+\sin ^{2} \frac{\hat{\phi}}{2}\right)}\right.} .
$$

The maximum synchrotron frequency is located at $\hat{\phi}_{\text {crit }} \approx$ $117^{\circ}$ (see also Fig. 1) with the numerical value given by $\omega_{s}^{\max } \approx 0.78 \omega_{s 0} / \sqrt{1+\Sigma}$. In Ref. [11] it was shown that if the bunch length $\phi_{m}$ exceeds $\hat{\phi}_{\text {crit }}$, Landau damping will be lost for frequencies close to $\omega_{s}^{\max }$ because of the vanishing derivative of the synchrotron frequency. Therefore this amplitude and the corresponding synchrotron frequency are called "critical." For small amplitudes $\left(\hat{\phi} \ll \hat{\phi}_{\text {crit }}\right)$ we obtain (see [7], p. 304, for $\Sigma=0$ )

$$
\frac{\omega_{s}}{\omega_{s 0}} \approx \sqrt{\frac{1}{1+\Sigma}} \frac{\pi}{2^{3 / 2}} \frac{\hat{\phi}}{K(1 / \sqrt{2})} .
$$

The synchrotron frequencies in single and double rf waves for $\Sigma=0$ are shown in Fig. 1 


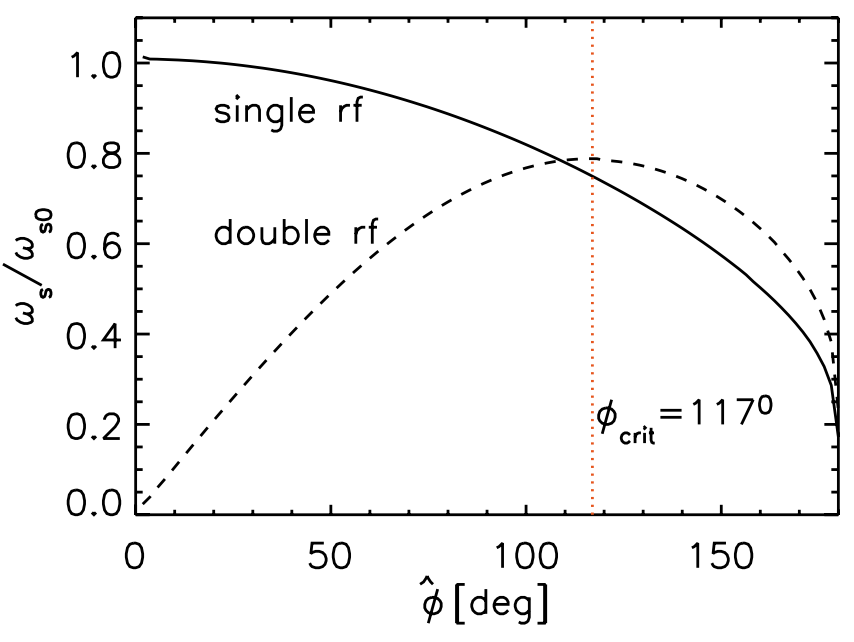

FIG. 1. (Color) Synchrotron frequency in single and double rf waves for $\Sigma=0$. The dotted vertical line represents the critical amplitude $\hat{\phi}_{\text {crit }}$ in a double rf wave.

\section{V. “RIGID” DIPOLE OSCILLATIONS}

Let $\phi_{c}$ be the position of the bunch center. If a matched bunch is rigidly displaced by the amount $\Delta \phi_{c}=\phi_{c}-\phi_{s}$ from the synchronous phase, the net force acting on the bunch center can be obtained from the rf force averaged over the bunch profile (see also Ref. [8])

$$
\ddot{\phi}_{c}=\omega_{s 0}^{2} \frac{1}{N} \int_{\phi_{m 1}}^{\phi_{m 2}} \frac{V_{\mathrm{rf}}}{V_{0}} \lambda\left(\phi-\phi_{c}\right) d \phi .
$$

Expanding the integrand for small displacements $\left(\Delta \phi_{c} \ll\right.$ $\pi$ ) yields the equation of motion for a harmonic oscillator with the dipole oscillation frequency

$$
\Omega_{c}^{2}=\omega_{s 0}^{2} \frac{1}{\left|u_{m}\right|} \int_{\phi_{m 1}}^{\phi_{m 2}}\left(\frac{V_{\mathrm{rf}}}{V_{0}}\right)^{2} d \phi
$$

For a stationary single rf wave we obtain

$$
\left(\frac{\Omega_{c}}{\omega_{s 0}}\right)^{2}=\frac{2 \phi_{m}-\sin 2 \phi_{m}}{4 \sin \phi_{m}-4 \phi_{m} \cos \phi_{m}}
$$

and for a double rf wave

$$
\begin{aligned}
& \left(\frac{\Omega_{c}}{\omega_{s 0}}\right)^{2} \\
& \quad=\frac{\frac{5}{4} \phi_{m}-\sin \phi_{m}-\frac{1}{2} \sin 2 \phi_{m}-\frac{1}{16} \sin 4 \phi_{m}+\frac{1}{3} \sin 3 \phi_{m}}{-2 \phi_{m} \cos \phi_{m}+\frac{1}{2} \phi_{m} \cos 2 \phi_{m}+2 \sin \phi_{m}-\frac{1}{4} \sin 2 \phi_{m}} .
\end{aligned}
$$

In the limit of short bunches one obtains for a single rf wave

$$
\frac{\Omega_{c}}{\omega_{s 0}}=\left(1-\frac{\phi_{m}^{2}}{10}\right)^{1 / 2}
$$

and for a double rf wave

$$
\frac{\Omega_{c}}{\omega_{s 0}}=\sqrt{\frac{5}{14}} \phi_{m}
$$

\section{LANDAU DAMPING OF DIPOLE OSCILLATIONS}

Landau damping is lost when the coherent frequency (here the dipole mode) is outside the band of incoherent synchrotron frequencies. The corresponding threshold intensity for the space charge induced loss of Landau damping can be obtained from

$$
\Omega_{c}=\omega_{s}^{\min } \quad \text { or } \quad \Omega_{c}=\omega_{s}^{\max },
$$

with $\omega_{s}^{\min }\left(\omega_{s}^{\max }\right)$ being the minimum (maximum) synchrotron frequency inside the bunch. The first case is, e.g., relevant for a single rf wave above transition (or in the case of a broadband inductive impedance below transition), where the single particle synchrotron frequencies are shifted upwards with increasing space charge. Here the threshold intensity is determined by the lowest synchrotron frequency. Below transition (or for an inductive impedance above transition) the second case applies. Figure 2 shows $\omega_{s}^{\max }$ and $\omega_{s}^{\min }$ together with $\Omega_{c}$ in single and double rf waves as a function of the space charge parameter below transition. The respective threshold values are marked with crosses.

For short bunches the threshold parameters can be obtained analytically. In a single rf wave we obtain, with $\omega_{s}^{\max }=\omega_{s 0} / \sqrt{1+\Sigma}$ for $m^{*}>0$,

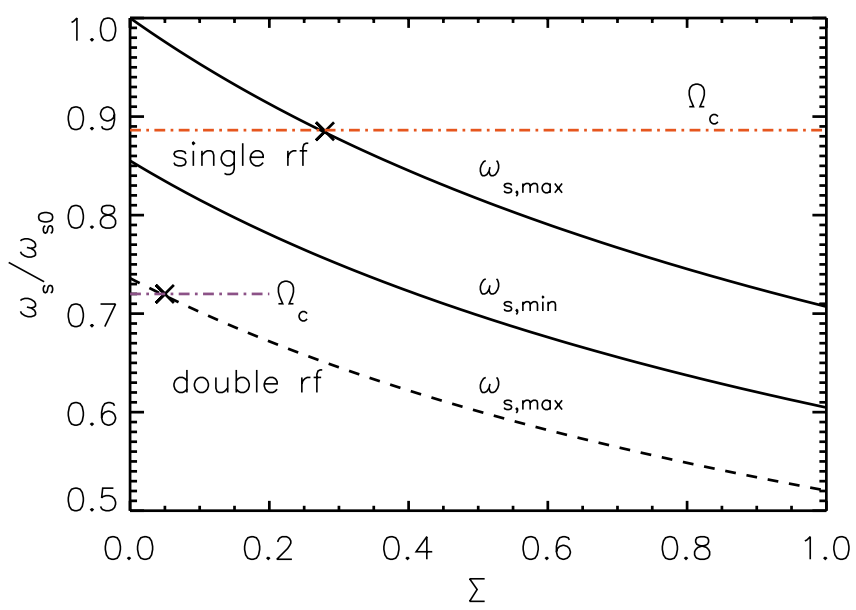

FIG. 2. (Color) Maximum and minimum synchrotron frequencies in single (solid lines) and double (dashed line) rf waves as a function of the space charge parameter. The bunch length is $\phi_{m}=90^{\circ}$. The two horizontal lines are the dipole oscillation frequencies in the single and in the double rf wave. The respective threshold values are marked with crosses. 


$$
\Sigma_{\text {th }}=\frac{1}{1-\frac{\phi_{m}^{2}}{10}}-1 \approx \frac{\phi_{m}^{2}}{10},
$$

and with $\omega_{s}^{\min }=\omega_{s}\left(\phi_{m}\right) / \sqrt{1-|\Sigma|}$ for $m^{*}<0$,

$$
\left|\Sigma_{\text {th }}\right|=\frac{\phi_{m}^{2}}{8}
$$

In a double rf wave $\omega_{s}^{\min }=0$ holds. The band of incoherent synchrotron frequencies in a double rf wave extends from 0 to $\omega_{s}^{\max }$. For positive $m^{*}$ and for a short bunch one obtains the constant threshold space charge parameter

$$
\Sigma_{\text {th }}=\frac{7}{20} \frac{\pi^{2}}{K^{2}(1 / \sqrt{2})}-1 \approx 0.005
$$

It is interesting to point out that for negative $m^{*}$ the criterion for Landau damping Eq. (35) is always fulfilled in the case of a double rf wave.

In Fig. $3 \Sigma_{\text {th }}$ is shown for stationary single and double rf waves. It can be seen that, in a single rf wave with increasing bunch length, the threshold space charge parameter increases $\sim \phi_{m}^{2}$. In a double rf wave and below transition Landau damping is lost at much lower space charge parameters ( $\Sigma_{\text {th }} \approx 0.005$ for short bunches) than in a single rf wave. Slightly above the critical bunch length $\phi_{\text {crit }} \approx$

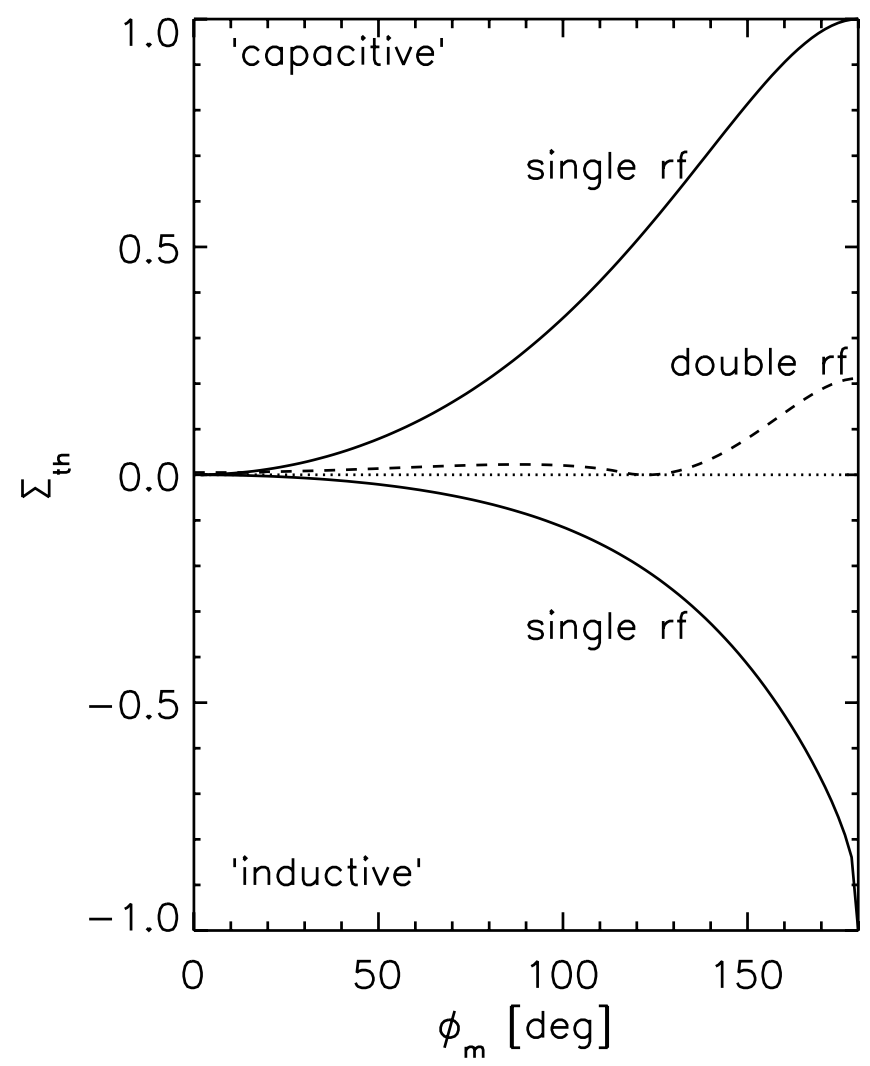

FIG. 3. $\Sigma_{\text {th }}$ as a function of the bunch length $\phi_{m}$ for stationary single and double rf waves above ("inductive") and below ("capacitive") transition. $117^{\circ}$, Landau damping is lost for all $\Sigma$. For bunch lengths exceeding this value $\Sigma_{\text {th }}$ increases again.

\section{OBSERVATIONS AND CURES IN THE SIS}

Persistent dipole oscillations of ion bunches are routinely observed in the GSI heavy ion synchrotron SIS $[10,15]$. This is due to the present lack of a rf phase control system in the SIS. If the energy of the injected beam from the UNILAC linear accelerator differs from the rf cavity frequency, the coasting beam is captured with a momentum offset. The resulting persistent dipole oscillations of the four $\mathrm{O}^{8+}$ bunches with $N=5 \times 10^{9}$ ions are shown in Fig. 4. For a bunch length of $\phi_{m} \approx 60^{\circ}$ after rf capture the corresponding space charge parameter is $\Sigma \approx 0.4$. This space charge parameter exceeds the threshold value (see Fig. 3), and therefore the observed persistent dipole oscillation can be attributed to the space charge induced loss of damping. From Fig. 3 we can also see that the installation of the new double rf system will lead to even lower threshold intensities for the loss of Landau damping. A digital phase control system will therefore be installed in the SIS [16] in order to damp longitudinal dipole oscillations during single and double rf operation. Another option would be an inductive insert [17] that compensates or, in the case of a double rf system, even overcompensates the space charge impedance. For exact compensation of the space charge impedance the nonlinear phase-mixing mechanism would be restored and coherent dipole oscillation would be damped, at the cost of an increased emittance.

It is important to point out that if Landau damping is lost for a mode, the beam is unstable with regard to every coupling impedance that drives this particular mode. The threshold space charge parameter can therefore be re-

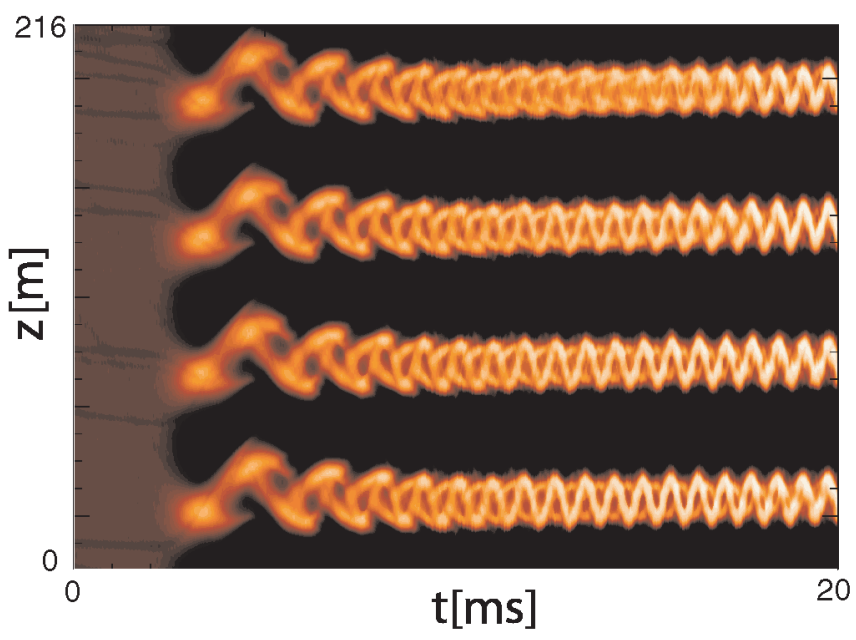

FIG. 4. (Color) Coherent dipole oscillations of four $\mathrm{O}_{18}^{8+}$ bunches with $N \approx 5 \times 10^{9}$ in the SIS at injection energy $(11.4 \mathrm{MeV} / \mathrm{u})$. The corresponding space charge factor is $\Sigma \approx 0.4$. 
garded also as an instability threshold parameter for the estimation of feedback requirements.

\section{BEAM TRANSFER FUNCTION}

In the previous sections the frequency of dipole oscillations as well as the intensity threshold for the loss of Landau damping were obtained in a non-self-consistent fashion. A more rigorous analytical approach would start from the linearized Vlasov theory. This approach was pursued in Ref. [11], where for $\Sigma=0$ the beam transfer functions (BTF) in single and double rf waves were calculated for $\mathrm{rf}$ amplitude and phase modulations. In the case of rf phase modulations the BTF in a single rf wave was defined in Ref. [11] as

$$
I_{1} \Delta \phi_{1}^{b}=I_{0} s_{1}(\Omega) \Delta \phi_{1}^{\mathrm{rf}},
$$

with the current amplitude $I_{1}$ at the main rf harmonic, the average current $I_{0}$, the harmonic rf phase modulation $\Delta \phi^{\mathrm{rf}}$, and the BTF $s_{1}(\Omega)$ as a function of the modulation frequency. If we assume a rigid bunch oscillation, the phase modulation of the beam current $\Delta \phi_{1}^{b}$ is equal to $\Delta \phi_{c}$, the phase displacement of the bunch center.

The BTF amplitudes for a single and double rf waves, $s_{1}(\Omega)$ and $d_{1}(\Omega)$, presented in Ref. [11] show pronounced maxima at $\Omega=\Omega_{c}$, with $\Omega_{c}$ being the rigid dipole oscillation frequency given in Eq. (32). In a double rf wave and for bunch lengths equal to or longer than $\phi_{\text {crit }}$, the BTF amplitude diverges for $\Omega=\omega_{s}^{\max }$. The infinite response for modulation frequencies $\Omega=\omega_{s}^{\max }=\omega_{\text {crit }}$ is due to the vanishing derivative of the synchrotron frequency inside the bunch. For those frequencies Landau damping is lost and within a linearized theory there is no other damping mechanism. From the analysis of the BTF it was concluded in Ref. [11] that long bunches in double rf waves are intrinsically unstable if a small perturbation is applied.

Using the matched elliptic distribution function together with the formalism presented in Ref. [11] it would be possible to calculate the BTF for bunches affected by space charge. However, even in the absence of space charge the linearized Vlasov theory gives results for long bunches and nonlinear $\mathrm{rf}$ that are at least questionable. Therefore we do not attempt to apply the linearized BTF formalism to bunches affected by space charge, but use a fully nonlinear simulation scheme.

\section{PARTICLE-IN-CELL (PIC) SIMULATION STUDY}

We employ a longitudinal particle-in-cell (PIC) simulation scheme $[18,19]$ that includes both the nonlinear external rf fields as well as the self-consistent space charge field calculated using a fast-Fourier transformation on a onedimensional grid with the grid spacing $\Delta z$. In order to reduce the effect of artificial noise on the particle dynamics, a large number of macroparticles per cell $\Delta z$ is used (typically larger than 1000) together with a quadratic spline interpolation. The number of cells is chosen as $N_{z}=$ 512 and the total number of macroparticles is $N_{\text {PIC }}=10^{6}$. The solver is part of a longitudinal beam dynamics code developed at GSI that incorporates different direct Vlasov solvers as well as the PIC solver used in this work. Both the Vlasov solvers, operating directly on $f(z, v)$, and the PIC solver, operating on macroparticles in $(z, v)$ space, use the same $2 \mathrm{D}$ and $1 \mathrm{D}$ grids and the same space charge routines. The solvers together with the space charge routine were benchmarked previously against various analytic examples (e.g., against echoes, see [20]), showing the ability of the solvers to resolve even the finest phase space informations. The code allows one to generate a macroparticle representation from an arbitrary distribution function $f(z, v)$ defined on the 2D grid in $(z, v)$ space. Therefore it is straightforward to load a matched elliptic distribution according to Eq. (10) in the PIC solver. Figure 5 shows a matched elliptic macroparticle distribution in a double $\mathrm{rf}$ wave.

\section{A. Simulation noise spectrum}

The Schottky noise from matched bunches in storage rings contains detailed information on the incoherent frequency spectrum as well as on the coherent mode spectrum. In ion storage rings the Schottky spectrum of coasting and bunched beams is an important experimental observable. It was shown in Ref. [3] that the simulation noise from a comparably low resolution PIC code $\left(N_{\text {PIC }}=\right.$ $5 \times 10^{4}$ ) reproduces very well the measured Schottky spectrum from cooled, very short $\left(\phi_{m} \lesssim 10^{\circ}\right)$ ion bunches

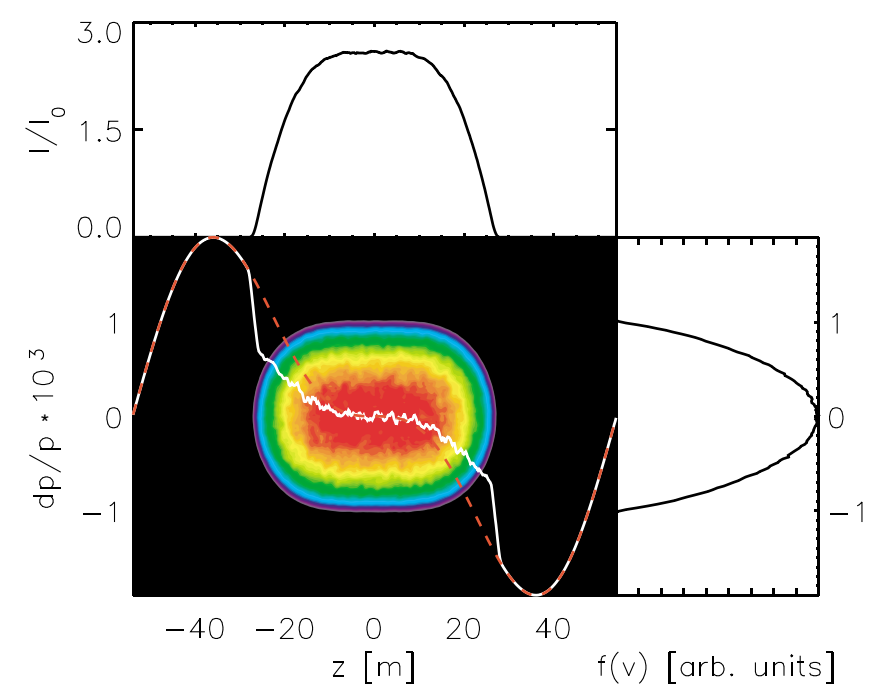

FIG. 5. (Color) Matched elliptic bunch distribution in a double rf wave. Macroparticle distribution, current profile, and velocity distribution from the PIC code for $\Sigma=1.0$ and bunch length $\phi_{m}=90^{\circ}$. The dashed red curve and the solid white curve represent the rf voltage profile and the total voltage profile, respectively. 
in a single rf wave. From the measured Schottky noise up to third order ("sextupole") bunched beam modes where resolved. From the simulation noise up to fourth order ("octupole") modes could be identified. The space charge induced splitting of the synchrotron satellites into incoherent and coherent lines was compared successfully with analytic formulas for linearized rf fields.

Here we will analyze the simulation noise from stationary, matched bunches affected by space charge and nonlinear rf fields (long bunches) using PIC simulations with high resolution. Figure 6 shows the simulation noise power spectrum from a matched elliptic bunch in a single rf wave as a function of the relative frequency $\Delta \omega=\omega-$ $h \omega_{0}$ divided by $\omega_{s 0}$ at harmonic $h=10$. The space charge parameter is $\Sigma=0$ and the bunch length $\phi_{m}=90^{\circ}$. Also shown is the momentum spread distribution of the bunch as a function of $\Delta \omega=-h \eta \beta_{0} c(\Delta p / p) / R$. Unlike the case of short bunches $\left(\phi_{m} \lesssim 60^{\circ}\right)$, only a small number of well separated, low order lines can be seen. Above $\Delta \omega \approx 4 \omega_{s 0}$ the lines are not equidistantly spaced. The shape of the "incoherent part" (above $\Delta \omega \approx 9 \omega_{s 0}$, without defined lines) of the frequency spectra and its width can be well described by the momentum spread distribution.

If we now "switch on" space charge $(\Sigma=1.0)$, the space charge induced splitting of low order lines can be observed (see Figs. 7 and 8). The positions of the first two lines in the spectrum can be reproduced very accurately by the maximum incoherent synchrotron frequency $\omega_{s 0} / \sqrt{1+\Sigma}$ and by Eq. (31) for the dipole mode frequency $\Omega_{c}\left(\phi_{m}\right)$. Also the second harmonic of the synchrotron frequency can be identified, whereas the second harmonic of $\Omega_{c}$ is still below the simulation resolution. Compared to the $\Sigma=0$ case, new lines outside the momentum spread distribution appear. These higher order

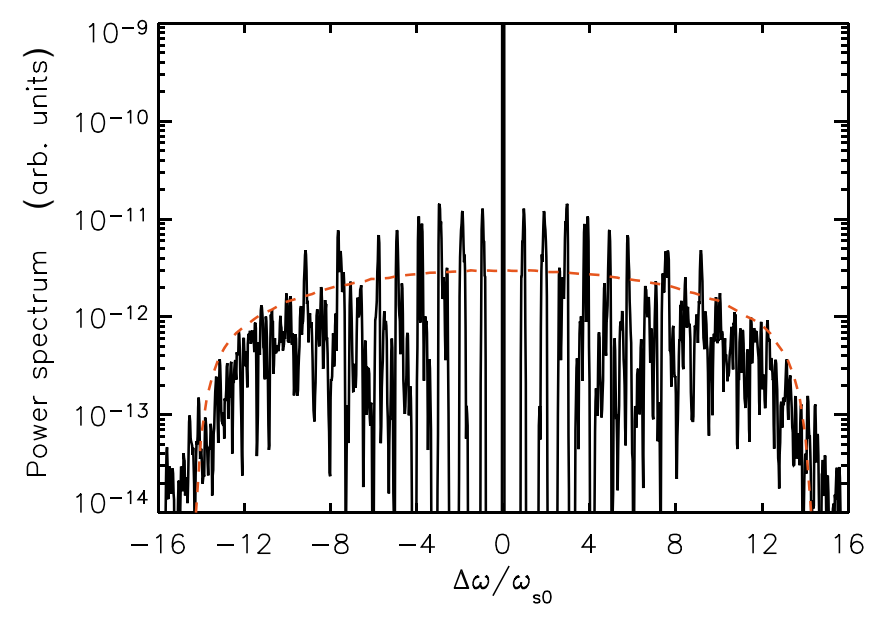

FIG. 6. (Color) Simulation noise power spectrum at harmonic $h=10$ from a matched bunch in a single rf wave. The space charge parameter is $\Sigma=0$ and the bunch length $\phi_{m}=90^{\circ}$. The dashed red line represents the momentum spread distribution of the bunch.

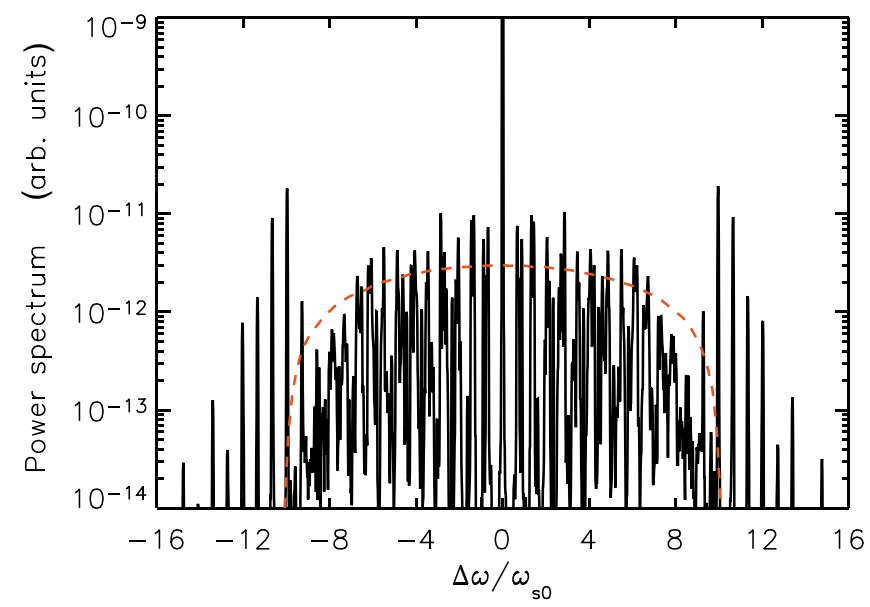

FIG. 7. (Color) Simulation noise power spectrum at harmonic $h=10$ from a matched bunch in a single rf wave. The space charge parameter is $\Sigma=1$ and the bunch length $\phi_{m}=90^{\circ}$. The dashed red line represents the momentum spread distribution of the bunch.

modes can be attributed to undamped space charge waves on the bunch. Because of the higher rf voltage required with space charge, the momentum spread distribution divided by the increased bare synchrotron frequency extends now to lower frequencies compared to the $\Sigma=0$ case.

The simulation noise spectrum from a bunch in a double rf wave is shown in Fig. 9 for $\Sigma=0$ and $\phi_{m}=90^{\circ}$. A general feature of the spectrum from a bunch in a nonlinear rf wave is the enhanced "incoherent" background noise. Peaks with identical partners on the positive (negative) frequency axis can hardly be identified, with the only exception being the dipole mode (see Fig. 10). The shape of the spectrum can be fitted with good accuracy by the momentum spread distribution. Switching on space charge $(\Sigma=1)$ results in a spectrum that exhibits pronounced

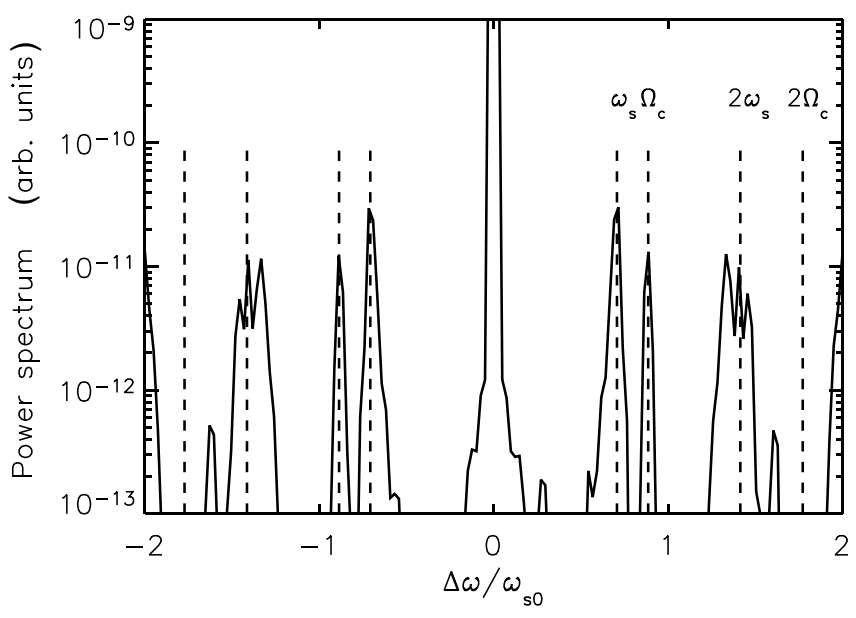

FIG. 8. Simulation noise power spectrum at harmonic $h=10$ from a matched bunch in a single rf wave. The space charge parameter is $\Sigma=1$ and the bunch length $\phi_{m}=90^{\circ}$. 


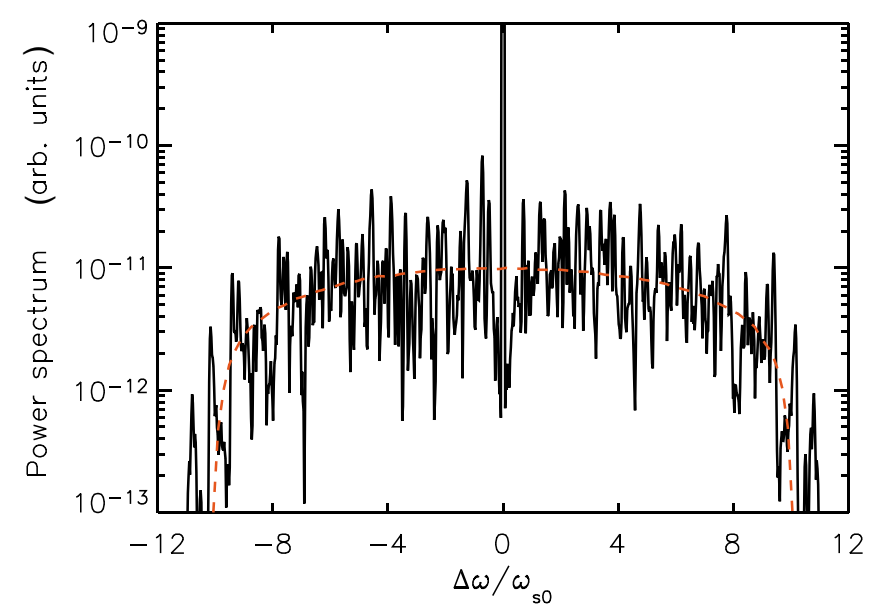

FIG. 9. (Color) Simulation noise power spectrum at harmonic $h=10$ from a matched bunch in a double rf wave. The space charge parameter is $\Sigma=0$ and the bunch length $\phi_{m}=90^{\circ}$. The dashed red line represents the momentum spread distribution of the bunch.

peaks at frequencies outside the momentum spread distribution (see Fig. 11). At low relative frequencies the only pair of lines that can be identified belongs to the dipole mode (see Fig. 12). The rest of the spectrum inside the momentum spread distribution remains incoherent. It is interesting to note that, in the presence of a frequency shifted high- $Q$ cavity impedance (below the threshold for the Robinson instability), one can observe the increase of the dipole mode lines at exactly $\pm \Omega_{c}$ in the noise spectrum also in a double rf wave.

It is important to point out that for long bunches exceeding the critical bunch length $\phi_{\text {crit }} \approx 117^{\circ}$ in a double rf wave we do not observe any noticeable changes relative to short bunches. In the presence of space charge the noise spectrum couples to the bunch motion and vice versa. The

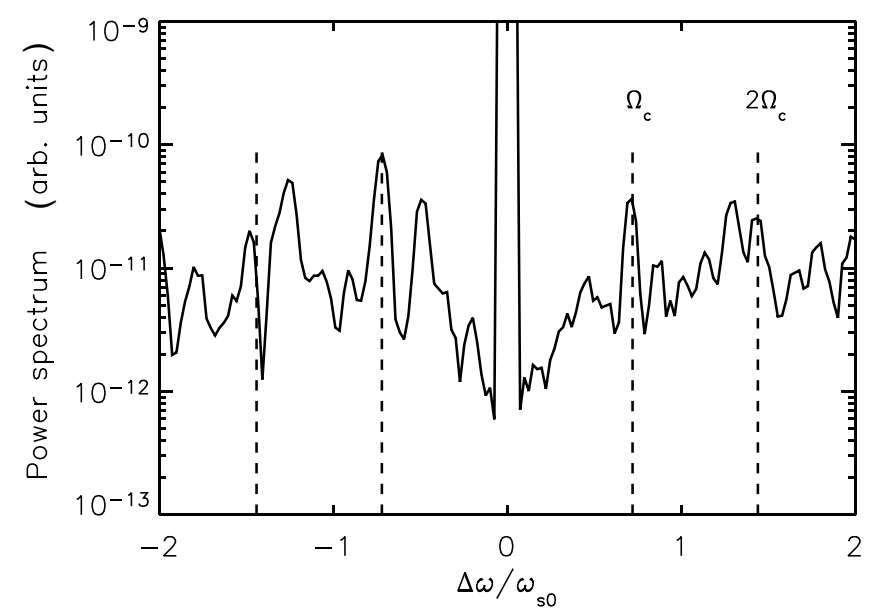

FIG. 10. Simulation noise power spectrum at harmonic $h=10$ from a matched bunch in a double rf wave. The space charge parameter is $\Sigma=0$ and the bunch length $\phi_{m}=90^{\circ}$.

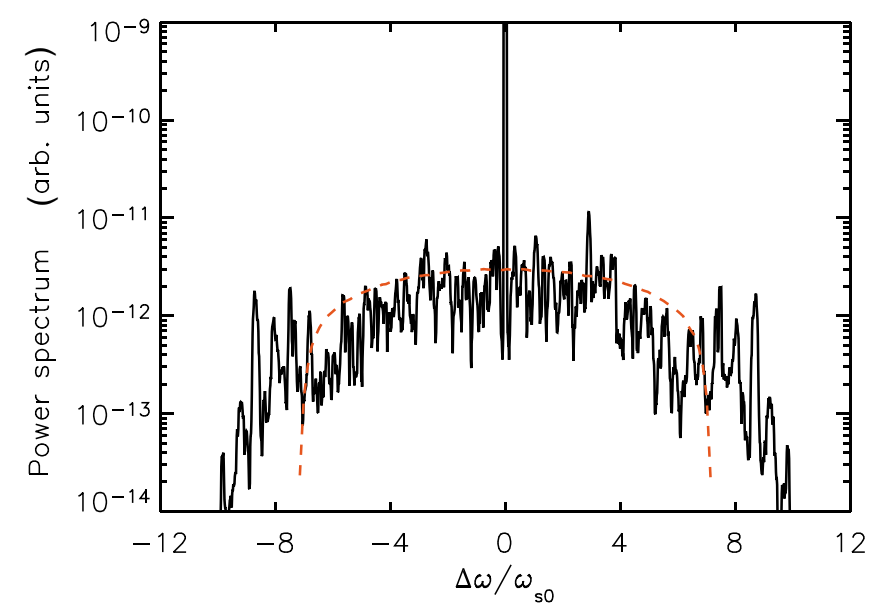

FIG. 11. (Color) Simulation noise power spectrum at harmonic $h=10$ from a matched bunch in a double rf wave. The space charge parameter is $\Sigma=1$ and the bunch length $\phi_{m}=90^{\circ}$. The dashed red line represents the momentum spread distribution of the bunch.

infinite beam transfer functions for $\Omega=\omega_{\text {crit }}$ suggest that in long bunches undamped modes could be excited from the noise, resulting, e.g., in an increased power spectrum.

\section{B. Beam response to a periodic perturbation}

The interpretation of the Schottky or simulation noise from long bunches in single or double rf waves can be quite complicated, as demonstrated in the foregoing section. In the case of matched bunches in a double rf wave, the noise spectrum contains mainly information on the incoherent bunch properties (momentum spread distribution). An alternative way of probing coherent properties of stable bunches is the application of weak rf phase or voltage modulations. In a PIC simulation code one obtains the "nonlinear" beam response as a function of the modula-

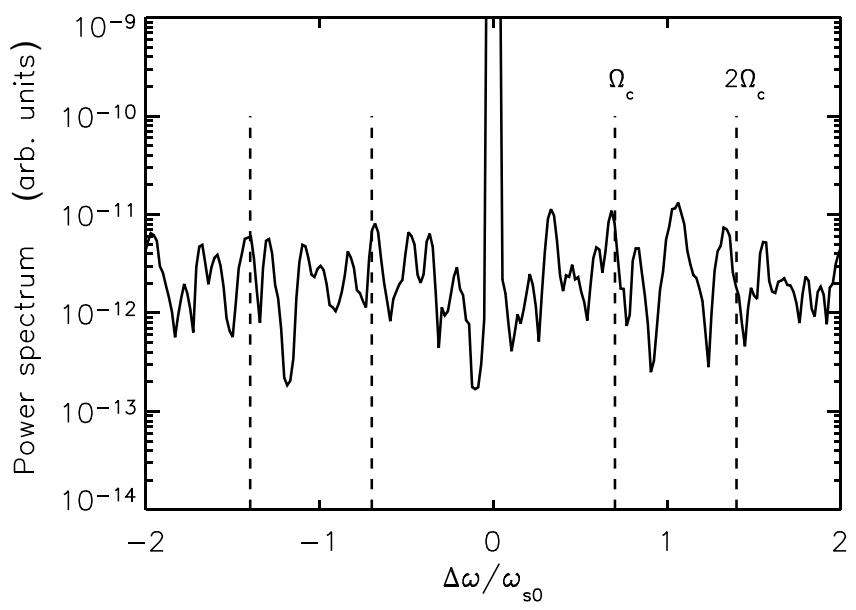

FIG. 12. Simulation noise power spectrum at harmonic $h=10$ from a matched bunch in a double rf wave. The space charge parameter is $\Sigma=1$ and the bunch length $\phi_{m}=90^{\circ}$. 
tion frequency. The results can be directly compared to the results from linearized Vlasov theory, provided that the code has a sufficiently low noise level. Here we apply a rf phase modulation $(\hat{\epsilon} \ll \pi)$ to matched, stationary bunches. The time-dependent rf voltage profile is given through

$$
V_{r f}(\phi)=V_{0}[\sin (\phi+\hat{\epsilon} \sin (\Omega t))+\alpha \sin (2 \phi)],
$$

with the modulation frequency $\Omega$ and the modulation amplitude $\hat{\epsilon}=\Delta \phi_{1}^{\text {rf }}$. As an example, Fig. 13 shows the bunch offset in a double rf wave as a function of time for $\Omega=\Omega_{c}, \Sigma=1.0$, and $\phi_{m}=90^{\circ}$. The rf phase modulation amplitude is chosen as $\hat{\epsilon}=0.1^{\circ}$. The amplitude of the driven dipole oscillation shows a modulation that occurs for resonant driving only. For driving frequencies $\Omega$ different from the rigid dipole mode $\Omega_{c}$ the amplitude saturates at a constant level.

In the following we define the beam response for given space charge parameter $\Sigma$, bunch length $\phi_{m}$, and modulation frequency $\Omega$ as the maximum bunch offset $\Delta \phi^{b}$ divided by the $\mathrm{rf}$ phase modulation amplitude $\Delta \phi_{1}^{\mathrm{rf}}$ at harmonic $h=1$. A beam response scan is obtained using many (about 100) simulation runs for different $\Sigma$ and $\Omega$ but fixed $\phi_{m}$. Figure 14 shows the beam response scan for a matched, stationary bunch $\left(\phi_{m}=60^{\circ}\right)$ in a single rf wave. It can clearly be seen that the maximum response is centered around $\Omega=\Omega_{c}$, with $\Omega_{c}$ given through Eq. (31).

Also one can observe that for $\Sigma>\Sigma_{\text {th }}$ (determined by the crossing point of $\omega_{\max }$ and $\Omega_{c}$ ) the maximum dipole amplitude increases. This can be explained by the space charge induced suppression of Landau damping, as explained in Sec. VI.

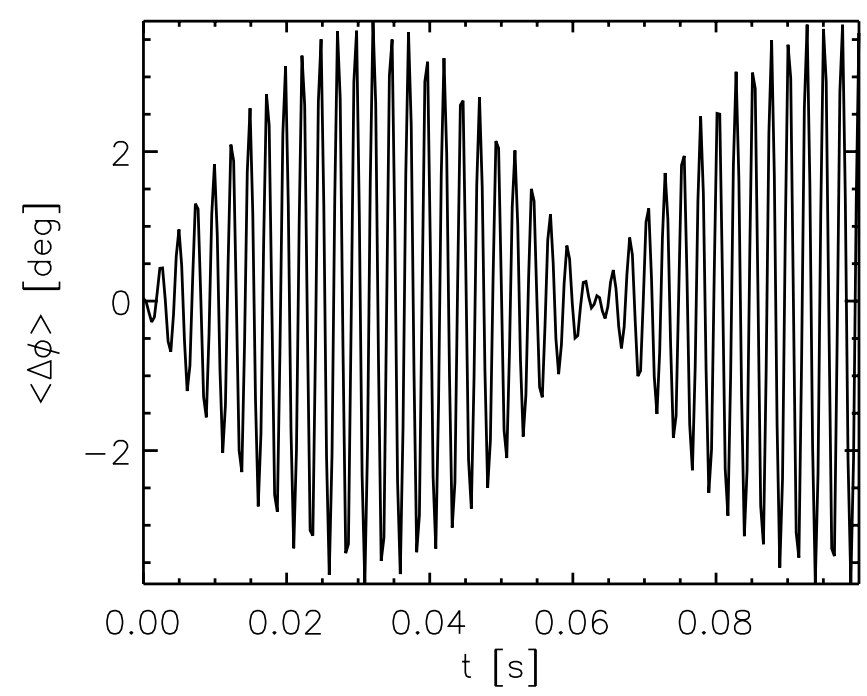

FIG. 13. PIC simulation of the bunch response to a phase modulation in a double $\mathrm{rf}$ wave. The modulation amplitude is $\hat{\epsilon}=0.1^{\circ}$, the bunch length $\phi_{m}=90^{\circ}$, the space charge parameter $\Sigma=1.0$, and the modulation frequency $\Omega=\Omega_{c}$.

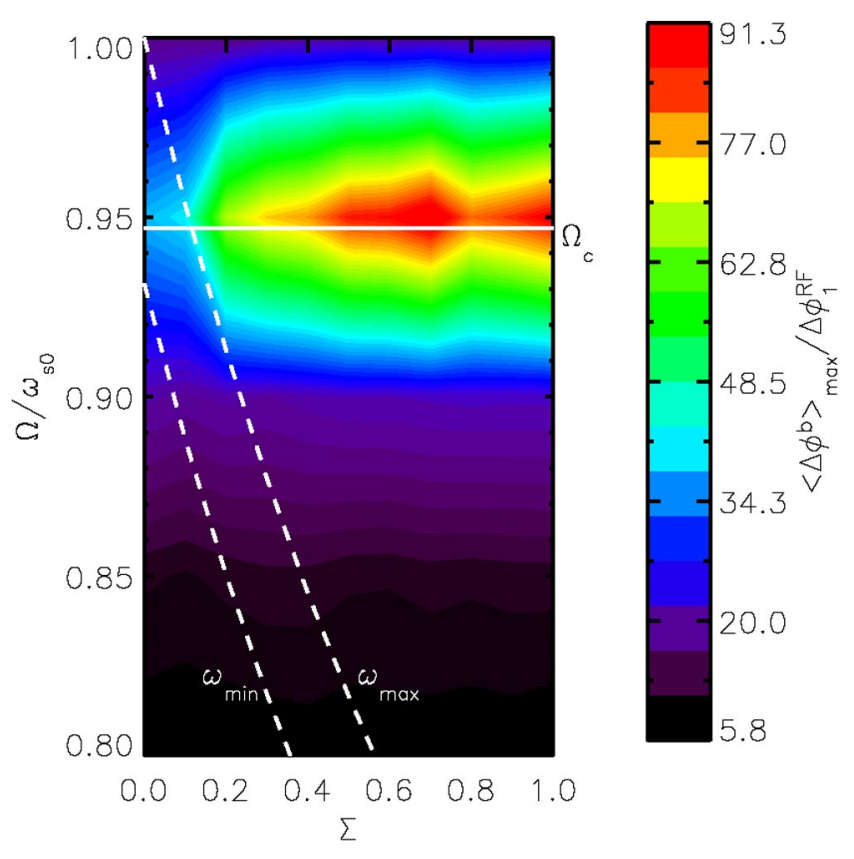

FIG. 14. (Color) Beam response scan for a matched, stationary bunch in a single rf wave. The color scale represents the maximum dipole amplitude divided by the rf phase modulation amplitude. The bunch length is $\phi_{m}=60^{\circ}$ and the rf modulation amplitude is $\hat{\epsilon}=0.1^{\circ}$. The white solid line is the rigid dipole mode frequency. The two dashed lines are the maximum and the minimum incoherent synchrotron frequencies.

The beam response in a double rf wave is shown in Fig. 15 for a bunch length of $\phi_{m}=90^{\circ}$. Here we observe that with increasing space charge the maximum dipole amplitudes occur at slightly lower frequencies relative to $\Omega_{c}$. For $m^{*}<0$ the beam response in a double rf wave decreases with increasing $|\Sigma|$ (see Fig. 16). As already pointed out in Sec. VI for $m^{*}<0$ the dipole frequency always remains within the band of incoherent synchrotron frequencies and Landau damping remains effective for all space charge parameters. This explains the weaker response seen in Fig. 16.

For long bunches, exceeding the critical bunch length $\phi_{\text {crit }} \approx 117^{\circ}$ in a double rf wave, we do not observe a pronounced dipole response for $\Omega=\omega_{\text {crit }}$, as would be expected from the BTF. Figure 17 shows the bunch response scan for a double rf wave and a bunch length of $\phi_{m}=145^{\circ}$. The maximum response occurs again for modulation frequencies $\Omega=\Omega_{c}$. For $\Omega=\omega_{s}^{\max }=\omega_{\text {crit }}$ we do not observe a noticeable dipole amplitude increase, but characteristic bunch shoulders around $\phi= \pm \phi_{\text {crit }}$ (see Fig. 18). It is interesting to note that similar shoulders on long bunches in a double rf wave were measured in the CERN SPS [21]. How these bunch shoulders affect and eventually restore Landau damping will be a topic of future work. Another noticeable change compared to the short bunch case (Fig. 15) is the bunch response for $\Omega>\Omega_{c}$ and $\Sigma \approx 0.6$. 


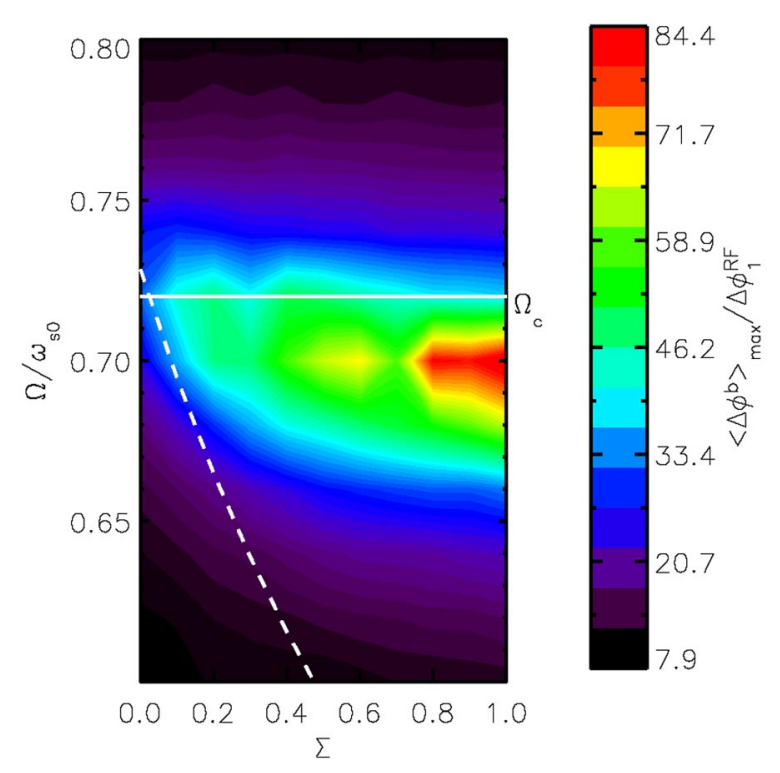

FIG. 15. (Color) Beam response scan for a matched, stationary bunch in a double rf wave. The color scale represents the maximum dipole amplitude divided by the rf phase modulation amplitude. The bunch length is $\phi_{m}=90^{\circ}$ and the rf modulation amplitude is $\hat{\epsilon}=0.1^{\circ}$. The white solid line is the rigid dipole mode frequency. The dashed line is the maximum incoherent synchrotron frequency.

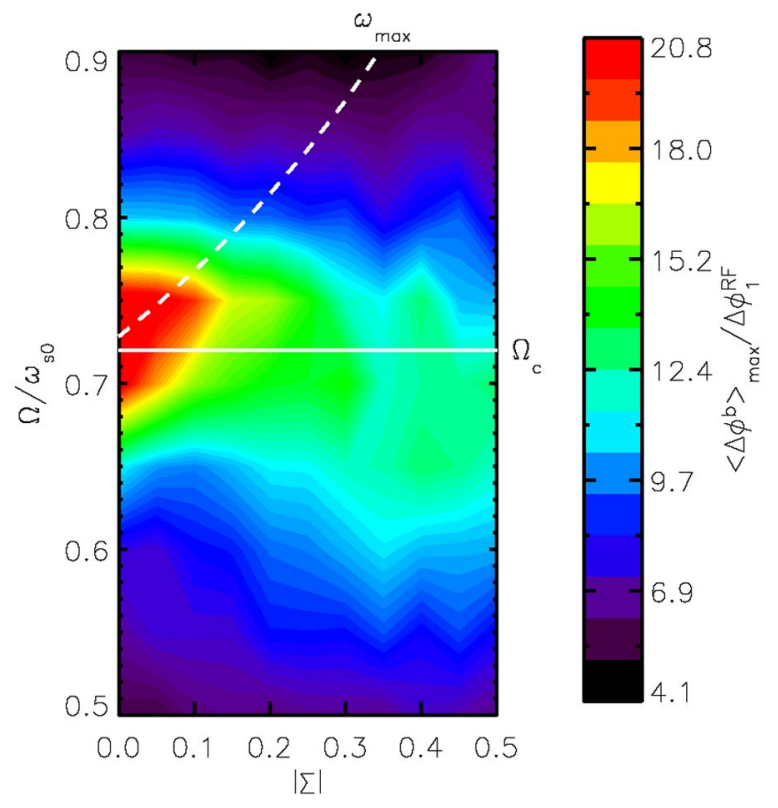

FIG. 16. (Color) Beam response scan for a matched, stationary bunch in a double rf wave and $m^{*}<0$ (above transition or inductive impedance). The color scale represents the maximum dipole amplitude divided by the rf phase modulation amplitude. The bunch length is $\phi_{m}=90^{\circ}$ and the rf modulation amplitude is $\hat{\epsilon}=0.1^{\circ}$. The white solid line is the rigid dipole mode frequency. The dashed line is the maximum incoherent synchrotron frequency.

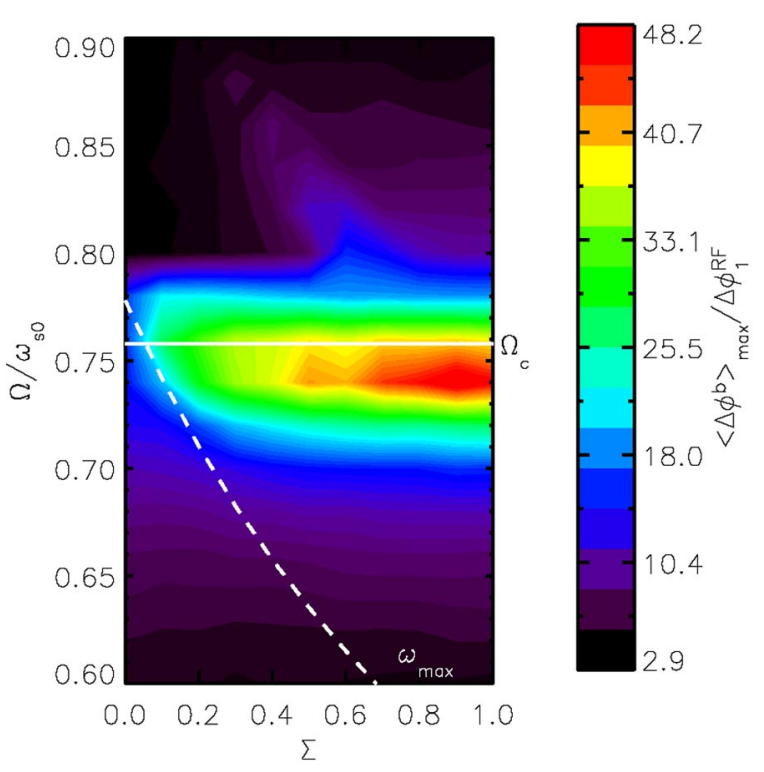

FIG. 17. (Color) Beam response scan for a matched, stationary bunch in a double rf wave. The color scale represents the maximum dipole amplitude divided by the $\mathrm{rf}$ phase modulation amplitude. The bunch length is $\phi_{m}=145^{\circ}$ and the rf modulation amplitude is $\hat{\boldsymbol{\epsilon}}=0.1^{\circ}$. The white solid line is the rigid dipole mode frequency. The dashed line is the maximum incoherent synchrotron frequency.

\section{FLATTOPPED BUNCHES}

The bunching factor in a double rf wave can be increased even further by diluting the bunch core and moving the particles into the bunch ends. If a double rf system cannot be employed, this technique can be used to increase the bunching factor in a single rf system. In practice, a coasting

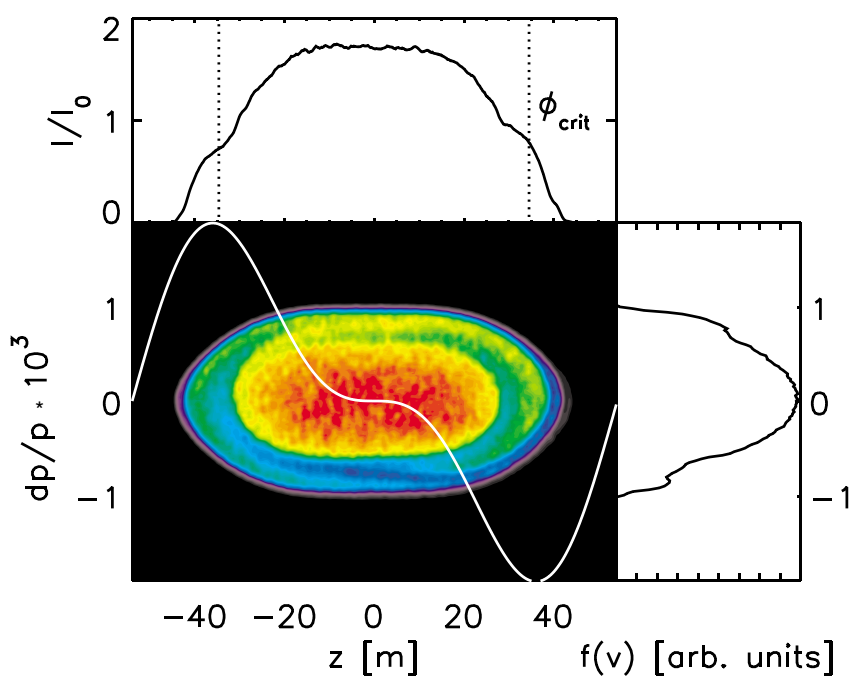

FIG. 18. (Color) Phase space density, bunch profile, and momentum distribution of a long bunch with $\phi_{m}=145^{\circ}$ and $\Sigma=$ 0.2 after about $50 \mathrm{rf}$ phase modulation periods with $\Omega=\omega_{\text {crit }}$. The modulation amplitude is $\hat{\boldsymbol{\epsilon}}=0.5^{\circ}$. 
beam with a double peaked momentum spread distribution has to be created prior to rf trapping [22]. Alternatively, it was shown in Ref. [23] that rf gymnastics using a second rf system can produce flattened bunches in a single rf wave. Ideally this reshaping leads to a flattopped bunch profile. Here we will discuss the stability of such ideally flattopped bunches in single and in double rf waves.

Mathematically a flattopped bunch can be created by subtracting two elliptic distributions with different bunch lengths [8]. One has to distinguish between "hollow" bunches and flattopped bunches. A flattopped bunch as defined here has a reduced phase space density and a corresponding constant current profile around the bunch center, whereas a hollow bunch should exhibit an unoccupied phase space area (a hole) around the center. Hollow bunches are unstable in the presence of space charge. Using a one-dimensional particle tracking simulation it was shown in Ref. [24] that hollow bunches "decay" through a dipole instability.

\section{A. Matched flattopped bunch distribution}

The distribution function for a flattopped bunch is obtained by subtracting two elliptic bunch distributions. For the subtracted ("inner") bunch parameters the index $h$ will used in the following. The index $m$ is kept for the main ("outer") bunch parameters. For the matched flattopped bunch distribution we obtain

$$
g(H)= \begin{cases}c_{1} \sqrt{H_{m}-\bar{H}}-c_{2} \sqrt{H_{h}-\bar{H}} & : H<H_{h} \\ c_{1} \sqrt{H_{m}-H} & : H_{h}<H<H_{m},\end{cases}
$$

with the Hamiltonian $H_{h}$ of the boundary particle of the inner bunch. For simplicity we specialize on symmetric bunches. The bunch limit for the main bunch is $z_{m}$, and for the inner bunch $z_{h}$. The line density for a flattopped bunch is constant for $-z_{h}<z<z_{h}$, and so the longitudinal space charge field of the main bunch is exactly canceled by the inner bunch. Therefore the particles inside the inner bunch see only the external rf field, and the value of the Hamiltonian for the particle on the inner bunch boundary is

$$
H_{h}=\frac{v_{h}^{2}}{2}=-\frac{q V_{0}}{m^{*} L} Y_{\mathrm{rf}}\left(z_{h}\right)
$$

The line density of a flattopped bunch is (see also Fig. 19)

$$
\lambda(z)=\left\{\begin{array}{lll}
\frac{N_{0}}{u_{m}}\left(Y_{\mathrm{rf}}\left(z_{h}\right)-Y_{\mathrm{rf}}\left(z_{m}\right)\right) & : \quad 0<z<z_{h} \\
\frac{N_{0}}{u_{m}}\left(Y_{\mathrm{rf}}(z)-Y_{\mathrm{rf}}\left(z_{m}\right)\right) & : \quad z_{h}<z<z_{m},
\end{array}\right.
$$

with the number of particles in the main bunch $N_{0}$. The total number of particles in the flattopped bunch is given through

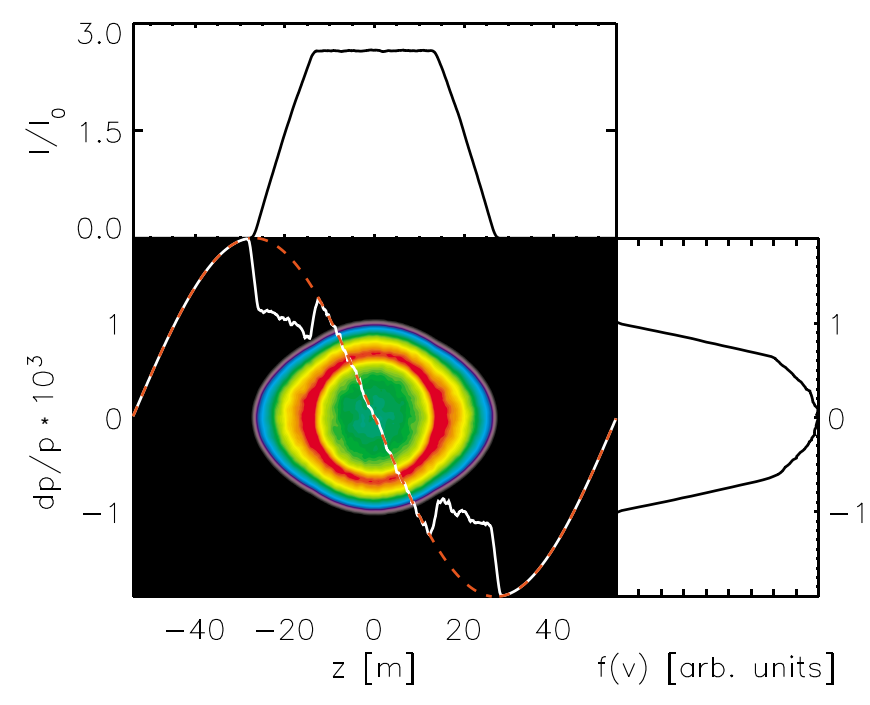

FIG. 19. (Color) Matched flattopped bunch distribution in a single rf wave. Macroparticle distribution, current profile, and velocity distribution from the PIC code for $\Sigma_{h}=0.4$, bunch length $\phi_{m}=90^{\circ}$, and $\phi_{h}=40^{\circ}$. The solid white line is the electric field including space charge. The dashed red line is the applied rf electric field.

$$
\begin{gathered}
N=N_{0}\left(1-\frac{u_{h}}{u_{m}}\right) \\
u_{h}=2 \int_{0}^{z_{h}}\left(Y_{\mathrm{rf}}(z)-Y_{\mathrm{rf}}\left(z_{h}\right)\right) d z .
\end{gathered}
$$

The bunching factor for a flattopped bunch is

$$
B_{f}^{h}=\frac{u_{m}}{u_{m}-u_{h}} B_{f},
$$

with the bunching factor $B_{f}$ for the main bunch. The space charge voltage amplitude $V_{0}^{h}$ for a matched flattopped bunch of half-length $z_{m}$ and an inner bunch half-length $z_{h}$ is

$$
V_{s 0}^{h}=V_{s 0}\left(1-\frac{Y_{\mathrm{rf}}\left(z_{h}\right)}{Y_{\mathrm{rf}}\left(z_{m}\right)}\right)
$$

with the amplitude $V_{s 0}$ for the main bunch only. The space charge parameter for a flattopped bunch is then defined as

$$
\Sigma_{h}=\frac{1}{V_{0} / V_{s 0}^{h}-1} .
$$

The potential seen by the particles inside the bunch can be written as

$$
Y(z)=\left\{\begin{array}{lll}
Y_{\mathrm{rf}} & : & 0<z<z_{h} \\
\frac{1}{\sqrt{1+\Sigma_{h}}}\left(Y_{\mathrm{rf}}(z)-Y_{\mathrm{rf}}\left(z_{h}\right)\right) & : & z_{h}<z<z_{m} .
\end{array}\right.
$$

In the following we will specialize on a single rf wave. Using Eqs. (21) and (22), together with Eq. (49), the synchrotron frequency for a flattopped bunch can be 
calculated. An analytic result can be obtained in the case of a linear rf field

$$
\frac{\omega_{s}}{\omega_{s 0}}= \begin{cases}1 & \frac{\pi}{2}\left[\left(\frac{\pi}{2}-\arcsin \frac{\phi_{h}}{\hat{\phi}}\right) \sqrt{1+\Sigma_{h}}+\arcsin \left(\frac{\phi_{h}}{\hat{\phi}} \sqrt{\frac{1+\Sigma_{h}}{1+\frac{\phi_{h}^{2}}{\hat{\phi}^{2}} \Sigma_{h}}}\right)\right]^{-1}: \quad 0<\hat{\phi}<\phi_{h} \\ & : \quad \phi_{h}<\hat{\phi}<\phi_{m} .\end{cases}
$$

From Eq. (30) we can calculate the rigid dipole mode frequency for a flattopped bunch as

$$
\left(\frac{\Omega_{c}}{\omega_{s 0}}\right)^{2}=\frac{2\left(\phi_{m}-\phi_{h}\right)-\left(\sin 2 \phi_{m}-\sin 2 \phi_{h}\right)}{4 \sin \phi_{m}-4 \phi_{m} \cos \phi_{m}-4 \sin \phi_{h}+4 \phi_{h} \cos \phi_{h}}
$$

and for a short bunch

$$
\frac{\Omega_{c}}{\omega_{s 0}} \approx\left[1-\frac{\phi_{m}^{2}}{10}\left(1+\alpha_{h}^{2}\right)\right]^{1 / 2}
$$

with $\alpha_{h}=\phi_{h} / \phi_{m}$. The dipole mode frequency of the flattopped bunch is reduced relative to the main bunch. This is because small amplitude (high frequency) particles were removed from the main bunch. Still for $\alpha_{h} \lesssim 0.6$ the dipole mode will always remain within the synchrotron frequency band. This is due to the fact that $\omega_{s}^{\max }$ in a flattopped bunch is always equal to $\omega_{s 0}$, independent of the space charge parameter $\Sigma_{h}$.

The exact synchrotron frequency as a function of $\hat{\phi}$ in a flattopped bunch with $\Sigma_{h}=1$ is shown in Fig. 20. One can see that for amplitudes smaller than the inner bunch length $\phi_{h}$, the frequency is given through the synchrotron frequency for $\Sigma=0$. For amplitudes approaching the bunch length $\phi_{m}$, the frequency comes close to the result for the

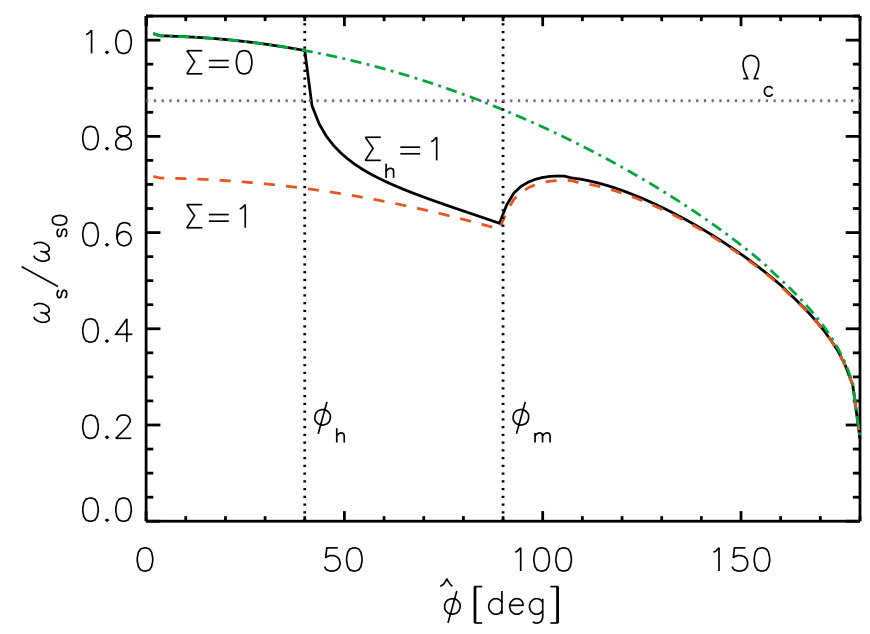

FIG. 20. (Color) The synchrotron frequency in a single rf wave as a function of the particle amplitude. The black solid line shows the result for a flattopped bunch with $\Sigma_{h}=1, \phi_{m}=90^{\circ}$, and $\alpha_{h}=0.45$. The red dashed line shows the result without the inner bunch for $\Sigma=1$, and the result for $\Sigma=0$ is represented by the green dash-dotted line. The horizontal line represents the dipole mode frequency $\Sigma_{c}$. The two vertical lines are the inner and the outer bunch length, respectively. main bunch and $\Sigma=1$. Here the dipole mode frequency is already well above the maximum synchrotron frequency for the main bunch.

In Fig. 21 the synchrotron frequency in a double rf wave is plotted. In a double rf wave the dipole mode frequency in the case of a flattopped bunch will slightly increase, because of small amplitude (low frequency) particles that are removed from the main bunch. The presence of the inner bunch will therefore lift the dipole mode above $\omega_{s}^{\max }$, even for $\Sigma=0$. Again for $\hat{\phi}<\phi_{h}$ the synchrotron frequency follows the result for $\Sigma=0$ and approaches the result for the main bunch with $\Sigma=1$ for $\hat{\phi} \gtrsim \phi_{m}$.

\section{B. Simulation study}

Within our simulation study we focus on the stability of matched flattopped bunches in different rf wave forms. For a range of space charge parameters and bunch length flattopped bunches in a single rf wave and below transition exhibit a space charge driven dipole instability. Figure 22 shows the growth of dipole oscillations in a flattopped bunch for $\phi_{m}=90^{\circ}, \alpha_{h}=0.45$, and $\Sigma_{h}=0.4$. A bunch stability scan over $\phi_{m}$ and $\Sigma_{h}$ is shown in Fig. 23. The relative length of the inner bunch is kept constant at $\phi_{h}=$ $0.45 \phi_{m}$. The color scale indicates the maximum dipole

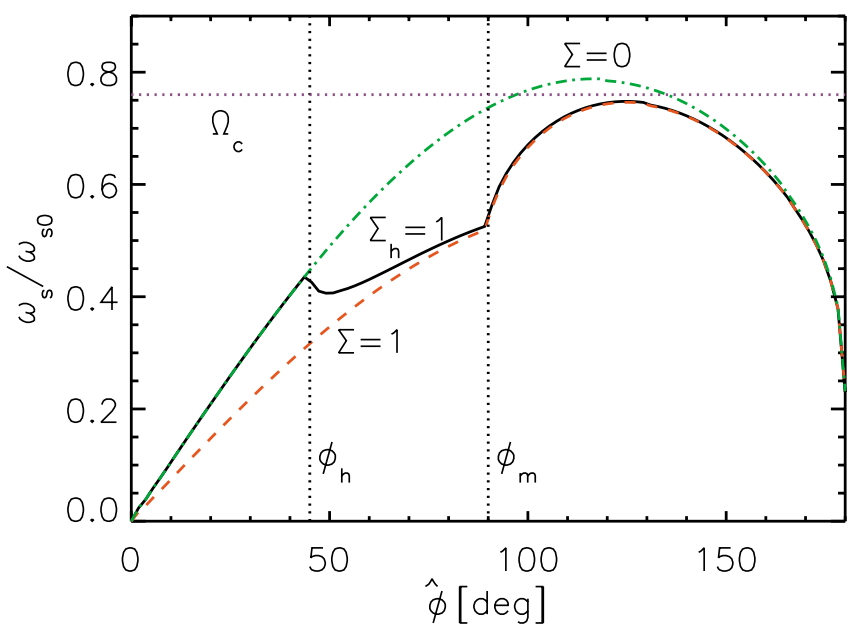

FIG. 21. (Color) The synchrotron frequency in a double rf wave as a function of the particle amplitude. The black solid line shows the result for a flattopped bunch with $\Sigma_{h}=1, \phi_{m}=90^{\circ}$, and $\alpha_{h}=0.45$. The horizontal line represents the dipole mode frequency $\Sigma_{c}$. The red dashed line shows the result without the inner bunch for $\Sigma=1$, and the result for $\Sigma=0$ is represented by the green dash-dotted line. The two vertical lines are the inner and the outer bunch length, respectively. 


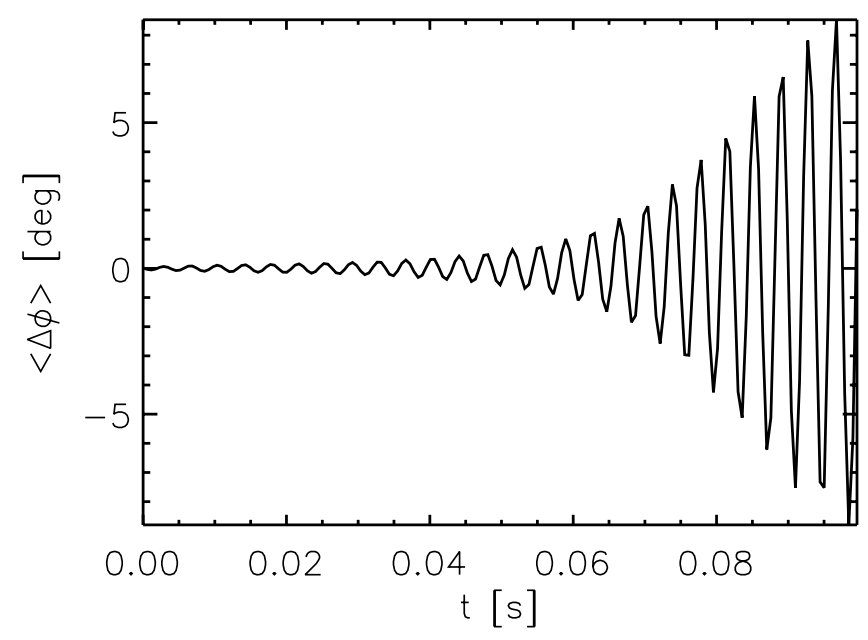

FIG. 22. Growth of the dipole mode amplitude for an initially matched flattopped bunch in a single rf wave. The bunch length is $\phi_{m}=90^{\circ}$, the length of the inner bunch $\phi_{h}=0.45 \phi_{m}$, and the space charge parameter $\Sigma_{h}=0.4$.

amplitude. It can be seen that there exists a well-defined area of unstable growth. Very short flattopped bunches $\phi_{m} \lesssim 45^{\circ}$ are stable. This is an indication that the instability has a nonlinear origin: Flattopped bunches in a linear rf field show no sign of unstable growth. For longer bunches and fixed $\phi_{m}$ the instability has a defined threshold intensity determined through a space charge parameter $\Sigma_{h}^{(1)}$. With increasing intensity there is a transition towards stable behavior at a space charge parameter $\Sigma_{h}^{(2)}$. The unstable region can be approximated by the two boundaries

$$
\Sigma_{h}^{(1,2)}=C_{1,2} \phi_{m}^{2}, \quad \text { with } C_{1} \approx 0.04, C_{2} \approx 0.2 \text {. }
$$

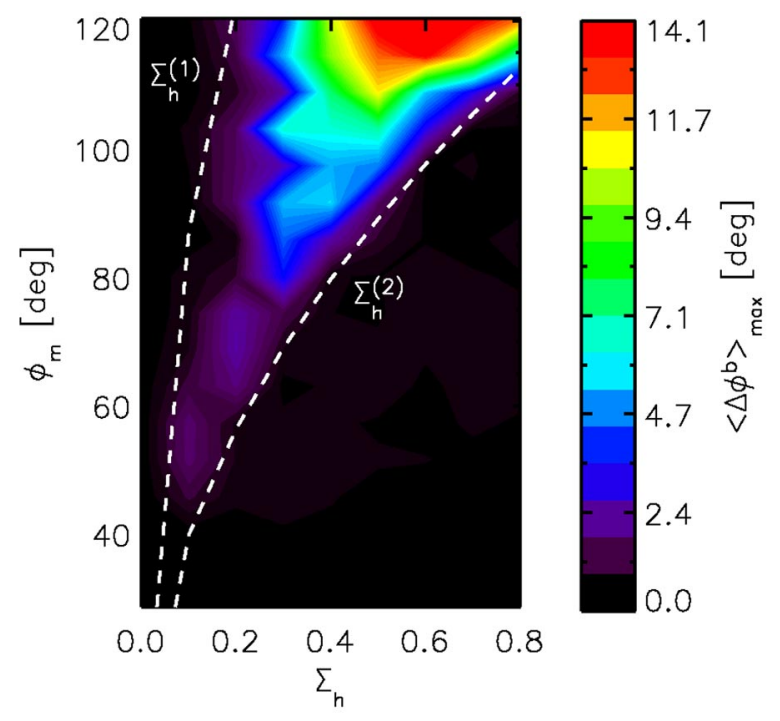

FIG. 23. (Color) Bunch stability scan for a matched flattopped bunch in a single rf wave. The color scale represents the maximum dipole amplitude. The length of the inner bunch is kept at $\phi_{h}=0.45 \phi_{m}$.

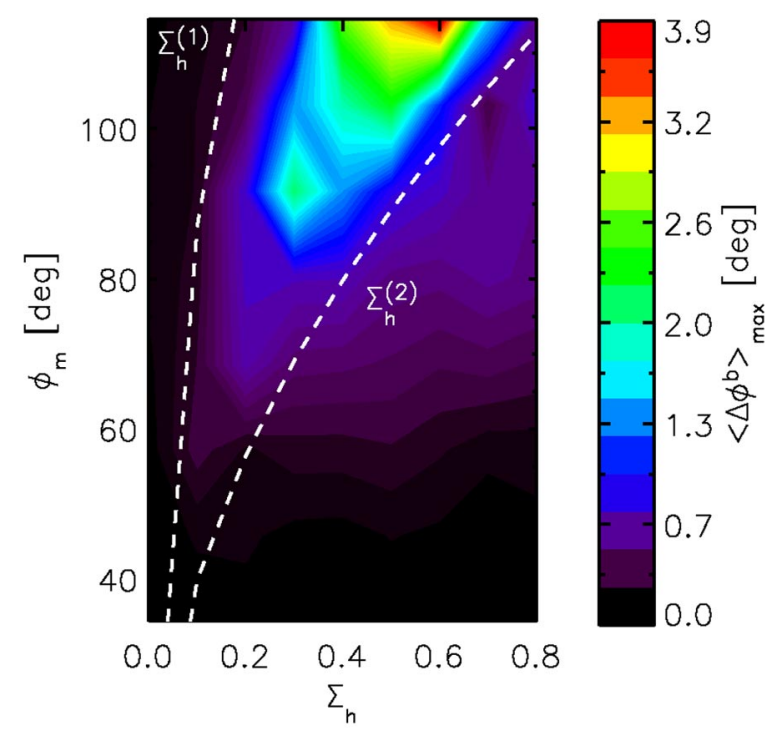

FIG. 24. (Color) Bunch stability scan for a matched flattopped bunch in a single rf wave. The color scale represents the maximum dipole amplitude. The length of the inner bunch is kept at $\phi_{h}=0.25 \phi_{m}$.

These two threshold curves can be compared with the Landau damping threshold in Eq. (36) for $\alpha_{h}=0$. It is interesting that both effects follow similar threshold laws. This can be interpreted such that the dipole instability results from the coupling of a band of incoherent synchrotron frequencies with the dipole mode. With decreasing size of the inner bunch the maximum unstable dipole amplitude decreases, but the boundaries of the unstable area remain the same. Figure 24 shows the bunch stability scan over $\phi_{m}$ and $\Sigma_{h}$ with the relative length of the inner bunch kept constant at $\phi_{h}=0.25 \phi_{m}$. For future studies it would be interesting to analyze whether a feedback system working on the dipole mode only could stabilize flattopped bunches in the unstable parameter region.

It is important to point out that, below transition, we observe no dipole instability in a double rf wave. As pointed out in the last section, in a double rf wave the dipole mode is lifted above all incoherent synchrotron frequencies and so there is no coupling. This is a possible explanation of the stability observed in our simulations. No simulation studies were performed for flattopped bunches above transition or for an inductive impedance.

\section{CONCLUSIONS}

In the framework of the elliptic distribution function we obtained analytic expressions for the synchrotron frequency, the rigid dipole oscillation frequency, and the threshold space charge parameter for the loss of Landau damping in single and double rf wave forms. It was shown that below transition energy and for long bunches the threshold in a double rf wave is much lower than in a single rf wave. On the contrary, we found that above 
transition Landau damping is always effective in a double rf wave.

We showed that the synchrotron frequency and the rigid dipole mode frequency can be identified from the "Schottky" simulation noise. Besides weak lines that can be attributed to the dipole mode, the noise spectrum from a bunch in a double rf wave is incoherent. The shape of the spectrum can be well approximated by the momentum spread distribution.

The effect of a weak rf phase modulation was studied using beam response scans. The maximum dipole amplitudes are excited for modulation frequencies tuned close to the rigid dipole mode. For bunch intensities exceeding the threshold space charge parameter for the loss of Landau damping, a strong increase in the maximum dipole amplitudes can be observed in both single and double rf waves.

We find that the infinite beam transfer functions obtained in Ref. [11] for long bunches in double rf waves do not lead to pronounced dipole amplitudes in our simulation studies. Instead we observe bunch shoulders, possibly restoring Landau damping. From this one can conclude that linearized Vlasov theory might not be adequate in order to predict the stability of bunches affected by nonlinear if fields. Besides simulation studies, an elaborate quasilinear Vlasov theory (see, e.g., Ref. [25]) could lead to more insight into damping and stabilization mechanisms in long bunches.

On the other hand, our work shows that the threshold space charge parameter $\Sigma_{\text {th }}$ for the dipole mode accurately predicts the observed strong increase of the bunch response in single and double rf waves. Preliminary simulation studies including resistive impedance sources show that $\Sigma_{\text {th }}\left(\phi_{m}\right)$ can approximate very well the instability thresholds in long bunches affected by space charge. Future work will also address the effect of space charge on high order modes in long bunches, where especially the quadrupolar and sextupolar modes will be studied. The experience at the Proton Synchrotron Booster, for example, shows that especially sextupolar modes can be excited in double rf waves [26].

As a last point, the stability of flattopped bunches was studied within our simulation code. For a well-defined range of space charge parameters and bunch lengths, flattopped bunches in a single rf wave exhibit a dipole instability. This instability occurs only in long bunches in a single rf wave. Flattopped bunches in a double rf wave are stable. As part of future work the comparison of our simulation results with beam transfer function calculation or other analytic approaches for flattopped bunches will be very useful.

\section{ACKNOWLEDGMENTS}

The authors would like to thank E. Shaposhnikova (CERN) for pointing out important experimental observa- tions related to double rf systems and for fruitful discussions. We are grateful to H. Damerau (CERN) for the careful reading of the manuscript and for his important comments. Fruitful discussions with the GSI rf group are also acknowledged.

[1] M. Reiser, Theory and Design of Charged Particle Beams (Wiley, New York, 1994).

[2] D. Neuffer, Part. Accel. 11, 23 (1980).

[3] I. Hofmann and G. Kalisch, Phys. Rev. E 53, 2807 (1996).

[4] G. Besnier, Ph.D. thesis, University of Rennes, 1978.

[5] P. Bramham, A. Hofmann, and P.B. Wilson, Report No. CERN-LEP/70-25, 1970.

[6] P. Hülsmann, O. Boine-Frankenheim, H. Klingbeil, and G. Schreiber, Report No. GSI-Acc-Note-2004-08-001, 2004.

[7] S. Y. Lee, Accelerator Physics (World Scientific, Singapore, 1999).

[8] A. Hofmann and F. Pedersen, IEEE Trans. Nucl. Sci. 26, 3526 (1979).

[9] A. N. Lebedev, At. Energ. 25, 100 (1968).

[10] O. Boine-Frankenheim, I. Hofmann, Y. Liu, and G. Rumolo, in Proceedings of the 2003 Particle Accelerator Conference, Portland, OR (IEEE, Piscataway, NJ, 2003), p. 2607.

[11] E. N. Shaposhnikova, Report No. CERN SL/94-19-RF, 1994.

[12] M. D’yachkov and R. Baartman, Part. Accel. 50, 105 (1994).

[13] M. Ellison et al., Phys. Rev. Lett. 70, 591 (1993).

[14] A. Al-khateeb, O. Boine-Frankenheim, I. Hofmann, and G. Rumolo, Phys. Rev. E 63, 026503 (2001).

[15] Y. Liu, GSI-Accelerator Report No. 2004-02-001, 2004.

[16] H. Klingbeil, IEEE Trans. Instrum. Meas. (to be published).

[17] M. A. Plum et al., Phys. Rev. ST Accel. Beams 2, 064201 (1999).

[18] C. K. Birdsall and A. B. Langdon, Plasma Physics via Computer Simulation (IOP, Bristol, 1991).

[19] R. W. Hockney and J. W. Eastwood, Computer Simulation using Particles (IOP, Bristol, 1994).

[20] A. Al-khateeb, O. Boine-Frankenheim, R. Hasse, and I. Hofmann, Phys. Rev. ST Accel. Beams 6, 014205 (2003).

[21] E. Shaposhnikova (private communication).

[22] A. Blas, S. Hancock, M. Lindroos, and S. Koscielniak, Report No. CERN-PS-2000-040-OP, 2000.

[23] C. Carli and M. Chanel, Report No. CERN-PS-2002-035AE, 2002.

[24] R .Baartman, F.W. Jones, S. Koscielniak, and G. H. Mackenzie, in Proceedings of the 1989 Particle Accelerator Conference, Chicago, IL (IEEE, Piscataway, NJ, 1989), p. 1196.

[25] A. A. Galeev and R. Z. Sagdeev, in Basic Plasma Physics, edited by A. A. Galeev and R. N. Sudan (North-Holland, Amsterdam, 1983), Vol. 1.

[26] A. Blas, S. Koscielniak, and F. Pedersen, Report No. CERN/PS 98-028/RF, 1998. 